\title{
Environmental conditions define the energetics of bacterial dormancy and its antibiotic susceptibility
}

\author{
L Mancini ${ }^{1, *}$ and T Pilizota ${ }^{1,+}$ \\ ${ }^{1}$ School of Biological Sciences, Centre for Synthetic and Systems Biology, University of \\ Edinburgh, Edinburgh, UK \\ *Currently at Department of Physics, Cavendish Laboratory, University of Cambridge, \\ Cambridge, UK \\ +Corresponding author:teuta.pilizota@ed.ac.uk
}

\begin{abstract}
Bacterial cells that halt growth but maintain viability and the capacity to regrow are termed dormant and have been shown to transiently tolerate high concentrations of antimicrobials. Dormancy has been seen in both tolerant and persister cells and is therefore of substantial clinical interest, as both can lead to infection recalcitrance and facilitate the development of antibiotic resistance. In this work, we look at dormancies induced by environmental cues that target different aspects of cell physiology by measuring the energy profiles they elicit in single dormant cells. Our simultaneous measurements of ATP concentration, proton motive force (PMF) and cytoplasmic $\mathrm{pH}$ reveal that dormant cells can exist in various energy states, offering a solution to the apparent mutual incompatibility of previous experimental results. We then test whether the energetic makeup is associated with survival to antibiotics of different classes. We find that for certain drugs growth arrest remains the dominant mechanism enabling survival, while for others, like ciprofloxacin, the most energetic cells remain almost untouched by the drug. Our results support a novel relationship between environment and drug susceptibility of dormant cells and suggest that knowledge of the conditions present at the infection site is necessary to design appropriate treatments.
\end{abstract}

Keywords:

\section{Introduction}

The global surge in bacterial antibiotic resistance in both clinical and environmental isolates highlights the need for a deeper understanding of bacterial survival strategies. Recent work Balaban et al. [2019], Brauner et al. [2016] has circumscribed clinically-relevant antimicrobial survival under antibiotic challenge to three main classes: resistance, tolerance and persistence. Resistant bacteria carry genetic mutations that allow them to thrive at antibiotic concentrations above the Minimum Inhibitory Concentration (MIC) Brauner et al. [2016]. Tolerant bacteria carry mutations too, but these only allow them to withstand antibiotics for a certain amount of time Brauner et al. [2016], Levin-Reisman et al. [2017]. Persistent bacteria are genetically undistinguishable from the susceptible population and develop a phenotypical form of tolerance Balaban et al. [2019], Brauner et al. [2016]. For both tolerant and persistent cells, leaving the growth cycle is a successful strategy for survival and dormancy is regarded as the silver bullet against a wide spectrum of antibiotics Lewis [2007], not only enabling infection recalcitrance Grant and Hung [2013], but also facilitating the development of resistance Levin-Reisman et al. [2017], Liu et al. [2020].

The mechanisms by which dormancy is attained, and how it subsequently leads to survival are multi-faceted and often poorly understood Rittershaus et al. [2013], Ronneau and Helaine [2019]. For example, the dormancy of cells in stationary phase is probably a result of several stimuli that could alone induce cessation of growth, such as starvation for multiple essential nutrients Pletnev et al. [2015] and the presence of quorum sensing molecules Lazazzera [2000]. The physiological state induced by bacteriostatic drugs is another example of dormancy, as these drugs cause growth arrest, but cells remain viable. Cells can stop growing in response to internal stimuli too, for example due to DNA damage 
Kreuzer [2013] or the accumulation of toxins in the cytoplasm Coussens and Daines [2016].

Even though dormancy can be achieved in multiple ways, these all lead to growth arrest. The natural connection between growth and metabolism has lead researchers to investigate the existence of a correlation between cellular energy (predominantly ATP Aedo et al. [2019], Braetz et al. [2017], Conlon et al. [2016], Leszczynska et al. [2013], Shan et al. [2017], Svenningsen et al. [2019] and PMF Grassi et al. [2017]) and survival capabilities of dormant cells. On one hand, stationary Staphylococcus Aureus was found to have high tolerance to ciprofloxacin and low ATP levels Conlon et al. [2016]. Similarly, reduced ATP levels in arsenate-poisoned Escherichia coli were linked with tolerance to ciprofloxacin and ampicillin Shan et al. [2017], and E. coli cells with high ATP levels were found more susceptible to $\beta$-lactams and fluoroquinolones Aedo et al. [2019]. On the other hand, Salmonella cells with reduced ATP levels due to deletion of the atp operon were more susceptible to ciprofloxacin Braetz et al. [2017] and ATP concentration was not low in ampicillin-tolerant stationary phase Leszczynska et al. [2013] and amino-acid starved Svenningsen et al. [2019] E. coli. These results, while appearing contradictory, could be consistent with the existence of dormant states with different energy levels and temporal profiles, and hence drug susceptibilities. As this would have relevant repercussions on treatment design, we investigated if it is the case by performing simultaneous single-cell measurements of multiple components of bacterial energetics in dormancies induced by different external stimuli. Specifically, we measured the cellular electrochemical gradient of protons (proton motive force, PMF), its $\Delta \mathrm{pH}$ component across the plasma membrane and the ATP concentration. PMF plays a fundamental role in cell physiology, powering exchanges across the plasma membrane Bradbeer [1993], Jahreis et al. [2008], Ramos and Kaback [1977], Wood [2015], including those mediated by multi-drug-efflux pumps Anes et al. [2015], and fuelling the production of ATP via $\mathrm{F}_{1} \mathrm{~F}_{o}$ ATP synthase, which can reversibly transform ATP and PMF into one another Mitchell [1961]. The intracellular portion of its $\Delta \mathrm{pH}$ component is actively controlled by cellular homeostatic processes as it regulates enzymatic activity and the stability of cellular structures Bearne [2014].

Our results show that the energetic states of cells that became dormant for different reasons are not equivalent and can range from growth-like to markedly reduced. In addition, we found that the energy profile and levels, characteristic for a given dormancy-inducing stimulus, influence the survival to antibiotics of different classes in a manner that appears energy-dependent. The importance of energetics could, to a first order approximation, be explained with the role of PMF/ATP on characteristic antibiotic accumulation. Our work portrays dormancy as a phenotypically complex state, a notion that we expect applies to similar states in other organisms, and that shows knowledge of the environmental conditions present at the infection site is important for the design of accurate treatment strategies.

\section{RESULTS}

Carbon starvation, bacteriostatic drugs and protonophores can induce instant dormancy. To investigate the energetics of bacterial dormancy we focused on E. coli and started by establishing a working definition of dormancy based on its most widely accepted hallmarks: growth arrest and capability to maintain viability. We note that this is different from Gefen et al. [2008], where in addition to being viable and non-growing, dormant cells also did not produce a reporter protein, probably indicating absence of protein production as a whole. We individuated three conditions that bacteria can encounter during infection: (a) carbon starvation, (b) high indole concentrations (a putative quorum sensing molecule produced by stationary phase cultures that has been shown to act as a protonophore at higher concentrations Chimerel et al. [2012], Kim and Park [2015], Krasnopeeva et al. [2019]) and (c) chloramphenicol treatment (a bacteriostatic antibiotic that stops protein synthesis Gale and Folkes [1953]). We assayed whether these induced a sharp growth halt and evaluated any viability losses. To assess the growth arrest, we grew cells in exponential phase for $\sim 15$ generations and then exposed them to carbon starvation and different concentrations of indole and chloramphenicol (SI Fig. 1A and B). To estimate growth we monitored bacterial cell numbers, which we measured via optical density (OD) over time. Fig. 1A shows that carbon starvation, $10 \mathrm{mM}$ indole or $200 \mu \mathrm{M}$ chloramphenicol cause immediate growth arrest, as indicated by the fact that OD remained constant. This, also indicates absence of lysis. However, OD is an indirect measure of cell number, with a proportionality that is linear only as long as cell size remains constant Stevenson et al. [2016], thus we checked that our candidate dormancies were not causing significant cell size changes during the switch between growth and dormancy, by monitoring the cells in a microscope (SI Video 1). Via microscopy we also confirmed that constant OD was not a result of matching growth 
and lysis rate. We achieved carbon starvation by moving the exponentially growing cells from growth media to the same media but with no carbon. Removing just the carbon source resulted in growth arrest, but the same was not possible by removal of the nitrogen source, perhaps indicating, as pinpointed by others before Kim et al. [2012], that nitrogen traces are present in common labware and can, at least to some extents, support bacterial growth (SI Fig. 1C). We next tested if the cells under our candidate dormancies were viable by washing the cultures into fresh medium and counting the colony forming units (CFU) over time, Fig. 1B. We found that the chloramphenicol treatment decreased viability by $40 \%$ after the first $30 \mathrm{~min}$, and down to 50\% over the $4 \mathrm{~h}$. Starvation had no effect on cell viability while indole at a concentration of $10 \mathrm{mM}$ reduced the number of survivors by $10^{6}$. The low number of survivors remained constant during the $4 \mathrm{~h}$ experimental window. At $10 \mathrm{mM}$, indole is a protonophore that causes PMF loss Krasnopeeva et al. [2019], which explains the significant loss of viability. Interestingly, the survival curve observed resembles the one typical of persistence Brauner et al. [2016], with the number of survivors, while low, remaining stable over time.
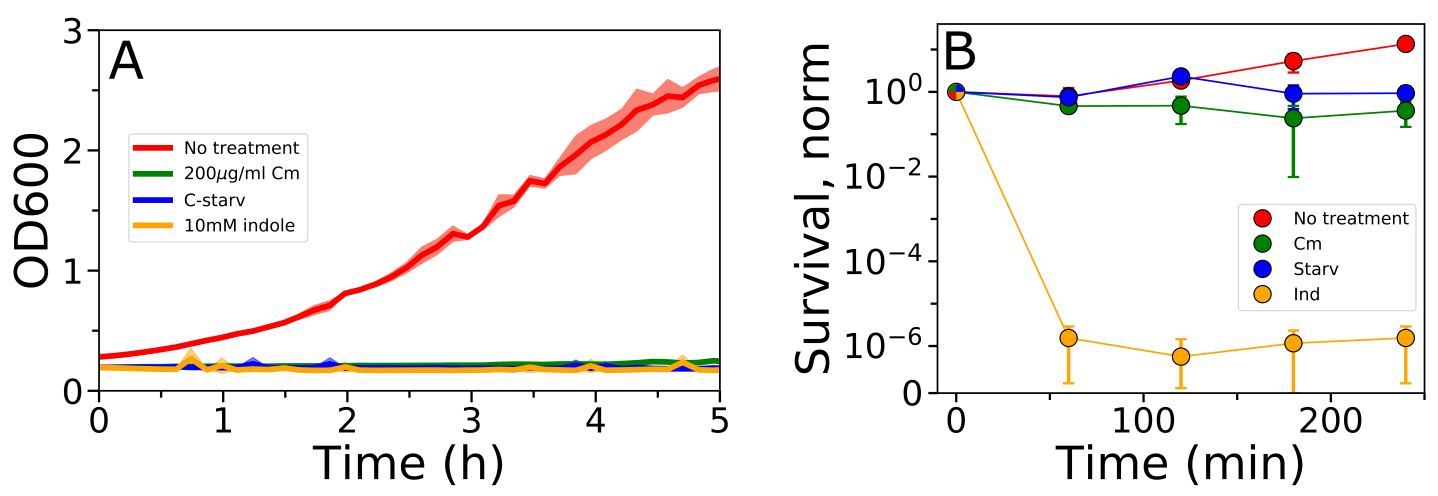

Figure 1. Individuation of different dormancies by monitoring cell growth and viability. a, addition of $200 \mu \mathrm{g} / \mathrm{ml}$ of chloramphenicol, removal of the carbon source and addition of $10 \mathrm{mM}$ indole sharply arrest growth of previously exponentially growing cells (collected at OD 0.3-0.4 prior to treatment and incubated in the plate reader at concentration that gives OD 0.2). The shaded area shows the standard deviation of 3 replicates and experiments were performed in a plate reader. $\mathbf{b}$, Cell viability under candidate dormancies identified in a assayed by counting colony forming units (CFUs). The error bars show the standard deviation of 3 independent replicates. 


\section{The PMF of dormant cells varies in a condition-dependent manner}

Having individuated dormancies that satisfied our definition, we next investigated their energy levels by measuring single cell PMF and one of its two components, the $\Delta \mathrm{pH}$ across the plasma membrane. We employed assays we previously developed Krasnopeeva et al. [2019], Mancini et al. [2020], Wang et al. [2019]. Briefly, we used bacterial flagellar motor speed as a proxy for relative PMF changes because it varies linearly with PMF Fung and Berg [1995], Gabel and Berg [2003]. To measure the motor speed we attached bacterial cells to the cover glass surface and the polystyrene beads to genetically modified, truncated flagellar filaments Krasnopeeva et al. [2019], Rosko et al. [2017], Ryu et al. [2000], Wang et al. [2019]. We measured the bead position, now rotated by the motor, with back focal plane interferometry thus gaining information on motor speed Krasnopeeva et al. [2019], Rosko et al. [2017], and we did so continuously throughout the treatment to include the transition into dormancy as well as the energy levels during dormancy. To simultaneously measure $\Delta \mathrm{pH}$ on the same cells, we constitutively expressed the fluorescent protein sensor pHluorin in the cell cytoplasm. We red its ratiometric signal every $90 \mathrm{~s}$, with illumination conditions that we had previously characterised as non-damaging to $E$. coli and with negligible photobleaching of the sensor Krasnopeeva et al. [2019]. To compute cytoplasmic pH values we compared fluorescence signal to a calibration curve we obtained from cells that cannot maintain $\Delta \mathrm{pH}$ (SI Fig. 2) Wang et al. [2019]. We performed our measurements on cultures that had grown for $\sim 25$ generations in fresh medium (SI Fig. 3), therefore reaching balanced exponential growth with a doubling time of 57 min, before transition into dormancy Harvey and Koch [1980], Maaløe [1966]. Fig. 2 shows different dormancies have characteristic energy profiles. In particular, cells treated with chloramphenicol underwent only a small PMF decline during the first 30 min of treatment, settling onto a relatively high, steady baseline thereafter (Fig. 2B). Cells experiencing carbon starvation settled onto an even lower baseline and the shift between the pre and post-treatment PMF levels occurred within 2-3 min (Fig. 2C). Lastly, cells treated with indole quickly lost their PMF, reaching motor speeds close to 0 within one or two minutes from the start of the exposure (Fig. 2D), and in agreement with previous measurements Krasnopeeva et al. [2019]. Internal pH shown in Fig. 2A showed similar trends for chloramphenicol treatment and carbon starvation. We were unable to measure the internal $\mathrm{pH}$ of cells treated with indole because the molecule interacts with pHluorin Wang et al. [2019]. However, because at the concentration used the molecule acts as a protonophore, we expect indole-treated cells to have no $\Delta \mathrm{pH}$. 

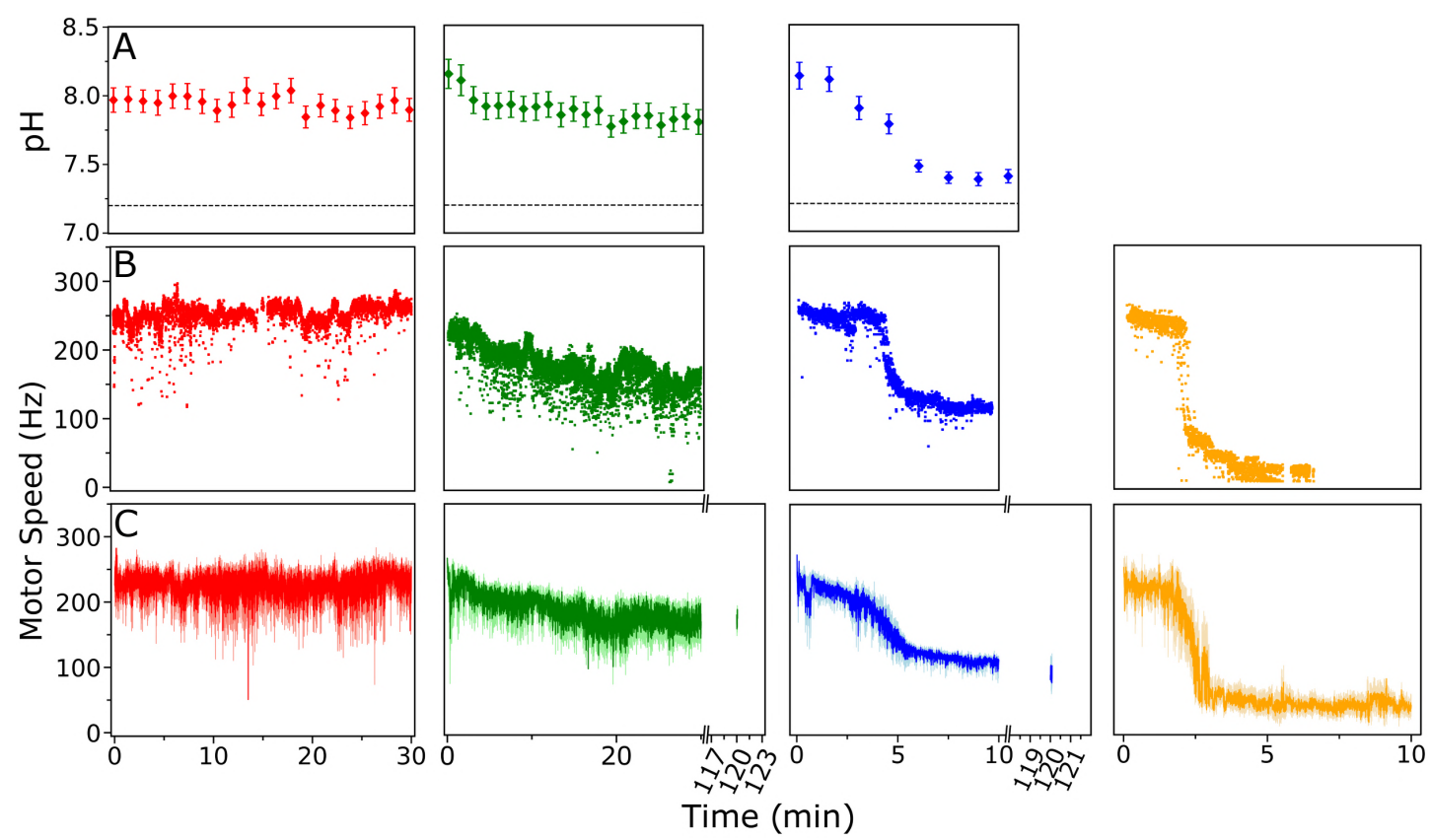

Figure 2. Different dormancies are not energetically equivalent. a, Average cytoplasmic $\mathrm{pH}$ and standard error for cells in growth medium (M63 with glucose shown in red), medium with $200 \mu \mathrm{g}$ chloramphenicol (green) and medium deprived of the carbon source (blue), when these are continuously supplied to the cells in a microscope flow-cell. Average values for each time point were obtained from 8 to 10 experiments for a total of 494 cells in M63 with glucose (media), 605 cells in the media with chloramphenicol and 594 cells in media with no glucose. Measurements are performed every $90 \mathrm{~s}$. Due to our flow slide design [Mancini et al., 2020] the switch between growth medium used during slide preparation and dormancy-inducing medium occurs 2 to $5 \mathrm{~min}$ from the beginning of the recording. b, Example motor speed trace (proportional to the PMF) from a single cell in the three conditions shown in $\mathbf{a}$, with the addition of the trace where the growth medium has been supplemented with $10 \mathrm{mM}$ indole (yellow). Measurements are performed at $10 \mathrm{kHz}$. c, Average motor speed and standard error (shaded area) of 10 cells in each condition (standard deviations are given in SI Fig. 4). In the case of chloramphenicol and carbon starvation we also assayed motor speed after $2 \mathrm{~h}$ incubation on 24 cells and 3 cells, respectively. It was not possible to find spinning motors after $2 \mathrm{~h}$ in the indole condition. 


\section{An improved QUEEN-based ATP sensor reveals ATP and PMF coupling during dormancy} Having measured PMF levels during dormancy we next focused on the ATP concentrations. Because we wished to measure time courses, ideally concurrently with single-cell PMF measurements, we chose the QUEEN ATP sensor Yaginuma et al. [2014]. However, the sensor suffers from poor signal intensity and requires exposure times of 1-2 $\mathrm{s}$ at short wavelengths, which can quickly lead to cell damage Krasnopeeva et al. [2019], Mancini et al. [2020]. Therefore, we focused first on optimising the sensor's expression level and signal-to-noise ratio. To improve the expression level we placed the sensor (QUEEN 2mM and QUEEN $7 \mu \mathrm{M}$ Yaginuma et al. [2014]) downstream of the strong constitutive promoter of the cytochrome C oxidase from V. Harveij Pilizota and Shaevitz [2012]. To improve the signal-to-noise ratio we expressed the QUEEN sensor with and without the His-tag. QUEEN has been tagged with a His-tag for protein purification, which is positioned in the proximity of the ATP sensing region and could have an effect on the protein's response to ATP (especially because in vivo ATP is commonly found in coordination with magnesium ions Gout et al. [2014]). We found that, particularly for QUEEN $7 \mu \mathrm{M}$, the removal of the tag produced an increase in signal intensity (Fig. 3A, SI Fig. 5A) and we named these brighter derivatives QUEEN 2mM* (Q2*) and QUEEN 7 $\mu \mathrm{M}^{*}(\mathrm{Q} 7 *)$. Because we could observe the cells expressing $\mathrm{Q} 7 *$ at $50 \mathrm{~ms}$ exposure times we selected this sensor variant for further in vitro characterization, which we performed on cells' lysates (see Methods). We first tested whether the His-tag removal affected the spectral properties of the protein and found them unchanged (SI Fig. 5B, SI Fig. 5D). Notably, the signal from Q7* from the lysates was high enough to render the spectral contribution due to other cellular components negligible (SI Fig. 5C). Next, we tested whether the sensor's response to ATP, i.e. its calibration, was sensitive to temperature. We observed a slight shift of the sensitivity range towards higher ATP concentration at higher temperature, indicating that the calibration should be performed for the specific temperatures at which the experiments are carried out (SI Fig. 5E). In addition, the sensitivity range of the Q7* sensor showed a significant shift towards higher ATP concentrations, compared to the original QUEEN $7 \mu \mathrm{M}$ (Q7) (SI Fig. 5E), making it suitable for performing ATP measurements in the physiological range of E. coli Yaginuma et al. [2014]. We then assayed the sensor's photostability, first focusing on whether photobleaching altered ATP calibration (SI Fig. 6). Again, using cell lysates for the in vitro characterization, we found that the shape of the calibration curve was not altered by illumination (SI Fig. 6D). Instead, for the whole curve, the correlation between ATP concentration and ratiometric fluorescence signal tended to shift with increasing illumination (SI Fig. 6D), which should be accounted for when time series measurements are performed. We also characterized the sensor in live cells. Because short wavelength illumination can lead to significant photodamage Krasnopeeva et al. [2019], we monitored bacterial PMF under different illumination frame rates. We identified that when illuminated every $7 \mathrm{~min}$ with 488 and $400 \mathrm{~nm}$ wavelengths for $20 \mathrm{~min}$ total, cells experience no photodamage (Fig. SI 7A-C). Next, we sought to assay sensors' in vivo photoeffects. We decouple the behaviour of the QUEEN sensor from cellular physiology by treating bacteria with ethanol, which drops the PMF Krasnopeeva et al. [2019], and we assume it thus limits any physiologically driven changes in the intracellular ATP concentration. We exposed the ethanol-treated cells to illumination at different frame rates observing, as expected, a frequency-dependent photobleaching at the $400 \mathrm{~nm}$ wavelength (SI Fig. 7D). Contrary to our observations with lysates, the signal from the $488 \mathrm{~nm}$ wavelength showed a behavior opposite to photobleaching (SI Fig. 7E), and overall, the 400/488 ratio decreased significantly with illumination (SI Fig.7F). The time-dependent increase in intensity observed in SI Fig. 7E under $488 \mathrm{~nm}$ illumination could be explained by photoactivation, a light-dependent red shift common among GFP and its derivatives Elowitz et al. [1997], Patterson and Lippincott-Schwartz [2002], or by a drop in the ATP concentration. The latter we deemed less likely given the ethanol treatment, but we further confirmed it by exposing otherwise untreated cells to the three dormancy-inducing conditions from Fig. 2. In Si Fig. 8 we show that in these cells, the sensor illuminated with either 400 and $488 \mathrm{~nm}$ wavelength behaves the same irrespective of whether or not bacteria have been exposed to different external conditions that we otherwise know cause changes in cellular energetics. We conclud that the increase in intensity observed under $488 \mathrm{~nm}$ illumination was most likely caused by photoactivation, and that given the sensors photoproperties, time series measurements should be replaced with an estimate of cellular ATP concentration at only one time point. For estimates of ATP concentrations in our dormant cells we chose as that time point the last PMF measurement from Fig. 2 (30 min for untreated and chloramphenicol-treated cells and $10 \mathrm{~min}$ for carbon-starved and indole-treated cells) after each dormancy induction. Fig. 3B shows that cells treated with chloramphenicol maintain constant ATP levels, while cells deprived of 
carbon or treated with indole show a reduction in ATP content, with the reduction being more marked in the indole-treated cells. The changes in ATP concentration obtained in the different conditions are qualitatively in line with our PMF and $\triangle \mathrm{pH}$ results indicating coupling of ATP and PMF during dormancy. We note that in the range of internal $\mathrm{pHs}$ we observed, the QUEEN sensors are insensitive to $\mathrm{pH}$ changes Yaginuma et al. [2014]. Our ATP concentrations are in the same order of magnitude of those reported by Yaginuma et al. [2014], although 2 to 5 times higher, probably due to different growth and imaging conditions.
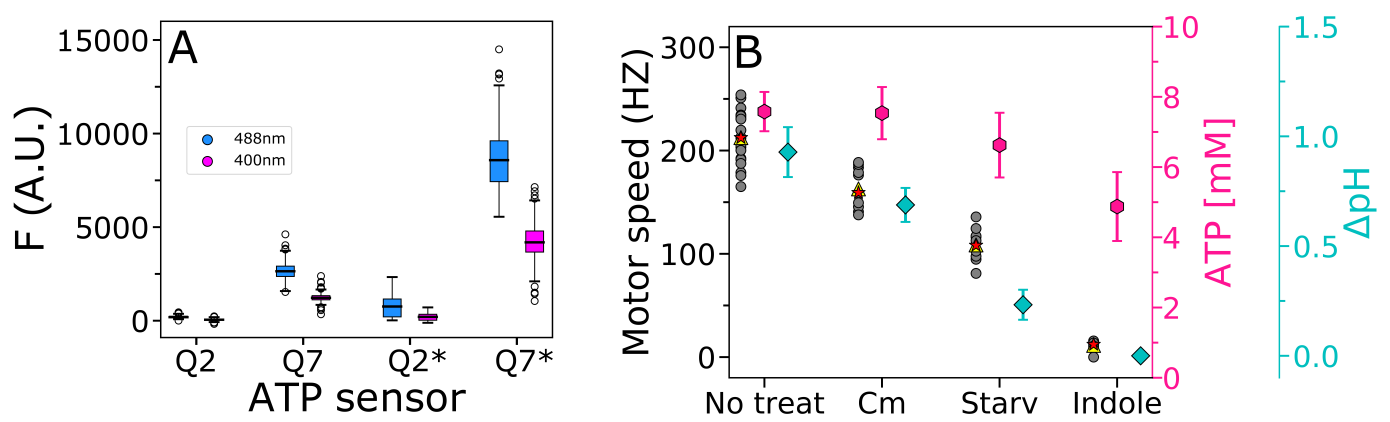

Figure 3. QUEEN $7 \mu \mathrm{M}^{*}$ shows improved signal intensity and can sense in E. coli's physiological range. a, Comparison of the signal intensity from the four sensors. Fluorescence values for the two wavelengths of interest for the QUEEN sensor (400 and $488 \mathrm{~nm}$ ) were obtained from 270 single cells expressing pWR20-Q2, 259 expressing pWR20-Q7, 194 expressing pWR20-Q2* and 315 expressing pWR20-Q7*. All cells were imaged in a tunnel slide (designed as given in Rosko et al. [2017]). The box plot shows lower and upper quartile and median value, with the whiskers indicating lowest and highest extremes. b, ATP and PMF of cells in different dormancies. Measurements were made after 30, 30, 10 and 10 min from the beginning of the treatment for non-treated, chloramphenicol-treated, starved and indole-treated cells, respectively. Grey circles indicate individual motor speeds under different conditions (median of $5 \mathrm{~s}$ long recording for each cell). Total of 20,11, 10, and 10 cells for non-treated, chloramphenicol-treated, starved and indole treated cells, respectively, are give. Red stars indicate the mean, and yellow triangles the median values. ATP concentration (pink) was measured in 1176 non-treated, 493 chloramphenicol treated, 633 starved and 793 indole treated individual cells. QUEEN $7 \mu \mathrm{M}^{*}$ sensor was used for the measurements. Cytoplasmic $\mathrm{pH}$ values (cyan) are from Fig. 2A. Because indole interacts with our $\mathrm{pH}$ sensor Wang et al. [2019] we could not measure it, but assumed $\Delta \mathrm{pH}$ of cells treated with indole is zero because indole is a protonophore Krasnopeeva et al. [2019]. For both ATP and pH the error bars show the standard error. 


\section{Dormant states with different energy levels show various degrees of susceptibility to representatives of different antibiotic classes}

Having measured different energetic profiles in different dormancies, we wondered if these influenced antibiotic susceptibility. This would have also been in agreement with previous findings showing that cellular energy levels play a role in antibiotic efficacy, e.g. by governing aminoglycoside drugs uptake rate Taber et al. [1987], fuelling multi-drug efflux pumps activity Anes et al. [2015] or other cellular response mechanisms such as DNA repair Kowalczykowski [1991], Kowalczykowski et al. [1994]. We exposed exponentially growing cells to the three dormancies, as in Fig. 2. After 30 min we exposed the dormant cultures to 5x MIC concentration of ciprofloxacin, a DNA damaging drug thought to be a substrate of multi-drug efflux pumps Piddock [2006] and kanamycin, an aminoglicoside that causes errors in protein translation Jelenc and Kurland [1984]. The MIC concentrations were obtained as explained in Methods and shown in SI Fig. 9. We assayed dormant cell survival for up to $4 \mathrm{~h}$, by withdrawing aliquots at regular intervals and performing CFU counting. For both of the antibiotics tested, chloramphenicol and carbon starvation-induced dormancy enabled cells to cope significantly better with antibiotic challenges than fast growing cells, confirming the protective role of dormancy. Cells treated with indole, which already represent a reduced portion of the population (Fig. 1B), showed poor survival outcomes, suggesting that to survive an antibiotic attack, dormant cells need to maintain a certain minimal level of energy. For both antibiotics tested, after $3 \mathrm{~h}$ treatment of indol dormant cells, one of the replicates showed unusually high survival. To confirm that the explanation is not highly variable lag times, we incubated indole plates for further $48 \mathrm{~h}$ observing no change in colony number. Instead, E. coli is known for forming clumps in liquid cultures in the presence of indole Beloin et al. [2008], which we could observe through flocculation of the cell pellet upon centrifugation of these samples. We conclude that these outliers are artifacts produced by a certain degree of dishomogeneity in the cell density. While for kanamycin we did not observe significant differences in survival between chloramphenicol and carbon starvation-induced dormancy, dormancy protection against ciprofloxacin showed energy dependence. After $4 \mathrm{~h}$ in the presence of the antibiotic, $28 \%$ of the chloramphenicol treated dormant cells survived, compared to the $4 \%$ of the carbon starved cells and the $0.1 \%$ of the growing ones.

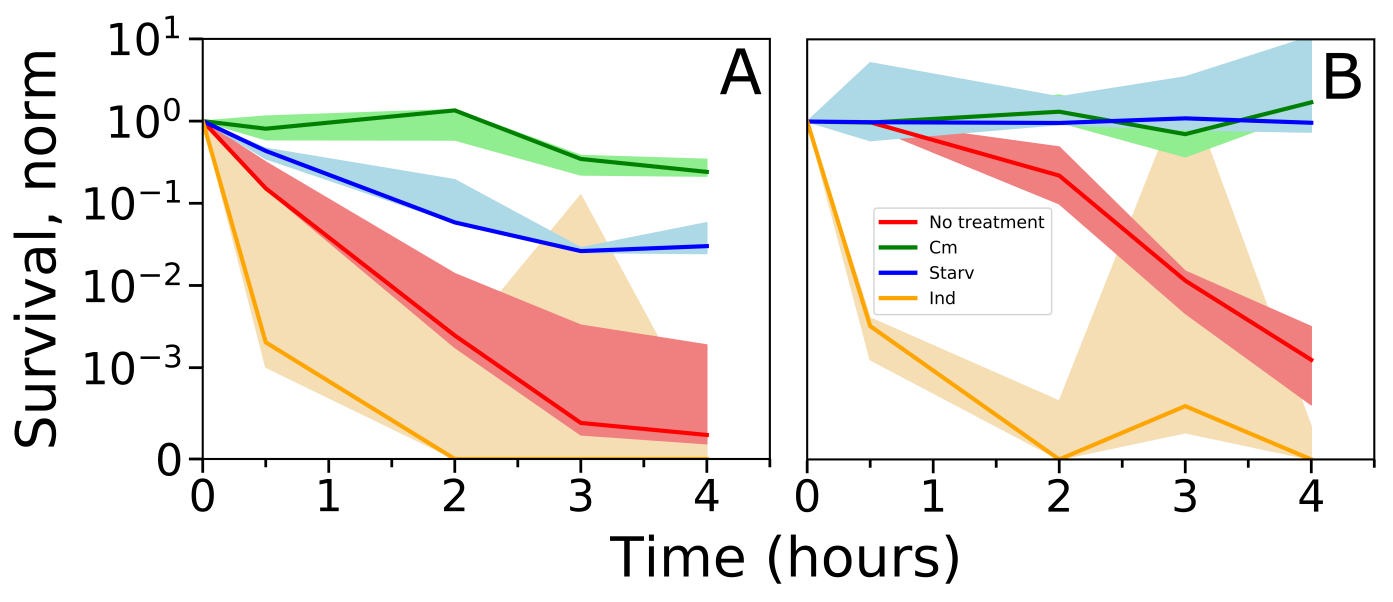

Figure 4. Survival of dormant cells to antibiotic treatment (at $5 \mathrm{x}$ MIC): $\mathbf{a}$, ciprofloxacin and $\mathbf{b}$, kanamycin. Cells that have gone dormant as a consequence of chloramphenicol treatment, carbon starvation and indole treatment are shown in green, blue and yellow. The antibiotics were added $30 \mathrm{~min}$ from initiation of dormancy, and CFUs were counted 30, 120, 180 and $240 \mathrm{~min}$ after the addition of antibiotic. In each experiment, counts under antibiotic treatment are normalised by the CFU count $30 \mathrm{~min}$ after initiation of dormancy (and before antibiotic addition). Solid lines show the median of 3 independent replicates and shaded areas the standard error of the mean. The same plot, but with solid lines showing averages is given in SI Fig. 10. 


\section{DISCUSSION}

Many antibiotics interfere with molecular targets that are essential during cell growth. Dormancy is thought to protect bacteria by making these drug targets non-essential. Yet the picture is probably more complex as not all non-growing cells are equally protected from antibiotics Gefen et al. [2008], and the mechanisms behind these differences remain an open question. In this work, we deployed an ATP concentration sensor, a cytoplasmic $\mathrm{pH}$ sensor and an assay for PMF measurements, to investigate the physiology of dormant cells at the single cell level. Rather than using an approach in which dormancy, and thus drug survival, emerge stochastically at a low frequency, we chose to induce dormancy a priori. This had several advantages. First, it allowed us to bypass the use of high-persistent genetic backgrounds like hipA7, and allowing us to work in effectively wild type backgrounds. Second, it gave us the freedom of testing various controlled conditions that can potentially occur at the infection site and directly link them to their effects on cells' phenotype. Third, because the whole cell population is exposed to dormancyinducing conditions, we could carry out low yield assays such as flagellar motor speed measurements yet obtain sufficient sample size. Our results show that cells that have gone dormant due to different external conditions settle onto markedly different energy profiles. In particular, across the conditions tested, energy levels range from growth-like to significantly reduced, demonstrating the existence of energy deprived dormant cells, but that not all dormant cells are low in energy. Our results show that during dormancy, ATP and PMF levels are coupled and so are PMF and cytoplasmic pH (and consequently the membrane voltage component).

Our results on different antibiotic susceptibility of dormant cells show several interesting points. Firstly, we could confirm that dormant cells outperform growing ones in terms of survival. However, our findings show that this is only true for those dormant states that maintained a minimum amount of PMF and ATP. The few cells that could survive indole treatment were more susceptible to the other antibiotics than growing cells, indicating that dormant cells do require a certain amount of energy to survive antimicrobial treatments. Secondly, the evidence we presented shows that, while for the aminoglycoside kanamycin growth arrest produces an almost complete protection, survival to the fluoroquinolone ciprofloxacin depends on the environmental conditions that induced dormancy. Intriguingly, cells treated with chloramphenicol that displayed only a minor reduction in PMF are almost unaffected by the antibiotic. Cells starved for carbon, that have suffered significant energy loss, perform an order of magnitude worse than their more energetic counterparts. Based on this correlation, we can speculate that energy availability during dormancy is an important factor in survival to ciprofloxacin. This is not only in agreement with previous work showing that stationary phase cells are susceptible to the drug Zeiler [1985], but it is also coherent with reports indicating that the main mechanisms of survival to ciprofloxacin involve drug-efflux pumps and the SOS response Tran et al. [2016], both energy-costly processes Blanco et al. [2016], Händel et al. [2013]. In the case of kanamycin the protection caused by growth arrest seems to mask any energy-dependent effects in our conditions. However, it is not excluded that these might emerge at higher drug concentrations or in dormant conditions that are less energetic than the carbon starvation we tested.

Taken together, our results show a previously unknown relationship between environmental conditions and drug susceptibility of dormant cells offering a mechanistic explanation for the different antibiotic efficacy. Furthermore, our results suggest that treatments based on the awakening of dormant cells by boosting metabolism may in some cases be counterproductive and that knowledge of the conditions at the infection site is necessary to appropriately fine tune treatments. We expect our conclusions to be relevant also for those variations in energy levels that emerge stochastically across cell populations and to be informative for dormancy and dormancy-like phenomena in other organisms.

\section{METHODS}

\section{Bacterial strains}

Experiments were carried out in the EK01 and EK07 strain Krasnopeeva et al. [2019]. This is an MG1655 background with the FliC-sticky mutation (EK01), allowing polystyrene beads to stick to bacterial flagella, and $\mathrm{pH}$ sensor pHluorin gene (EK07), both incorporated into the chromosome. For ATP concentration measurements, the EK01 strain was transformed with the plasmid pTP20-QUEEN7* that expresses the QUEEN $7 \mu \mathrm{M}^{*}$ sensor downstream of a strong constitutive promoter as shown in SI Fig. 11. As in Mancini et al. [2020], the plasmid was constructed by PCR amplification of the backbone from plasmid pWR20 Pilizota and Shaevitz [2012] and the sequence containing Q7*. Later steps included purification 
of the PCR products followed by restriction with the restriction enzymes AvrII and NotI (NEB, UK) and ligation with the T4 DNA ligase (Promega, UK). Chemically competent cells were transformed with the ligation mixes and resulting strains confirmed with colony PCR and sequencing. A map of the plasmid pTP20-QUEEN7* and the primers are given in SI (SI Fig. 11 and SI Table 1). All the strains used in the study are summarized in the SI Table 2.

\section{Bacterial growth conditions}

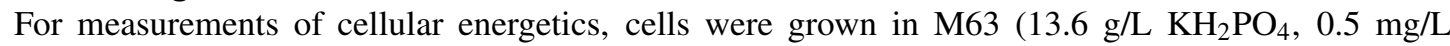
$\mathrm{FeSO}_{4} \cdot 7 \mathrm{H}_{2} \mathrm{O}, 0.5 \mathrm{~g} / \mathrm{L} \mathrm{MgSO} \cdot \cdot 7 \mathrm{H}_{2} \mathrm{O}, 1.27 \mathrm{mg} / \mathrm{L}$ Thiamine, $2.64 \mathrm{~g} / \mathrm{L}\left(\mathrm{NH}_{4}\right) 2 \mathrm{SO}_{4}$ and $0.5 \%$ w/v Glucose) to balanced growth at $37^{\circ} \mathrm{C}$ with shaking at $220 \mathrm{rpm}$. Balanced growth was achieved by inoculating single colonies into LB medium ( $0.5 \%$ Yeast Extract, $1 \%$ Bacto tryptone, $0.5 \% \mathrm{NaCl}$ ) for 3 to $4 \mathrm{~h}$. Cells from such culture were grown to OD 0.3-0.4 in M63 from a starting dilution of 10 ${ }^{-7}$. In the case of the EK01-pTP20-Q7* strain, $50 \mu \mathrm{g} / \mathrm{ml}$ of kanamycin was added to the medium.

Optical density readings in the presence of various concentrations of indole and chloramphenicol or in carbon deprived medium were obtained in a Spectrostar Omega microplate reader (BMG, Germany) using a flat-bottom 96-well plate that was covered with a lid during the experiments (Costar, UK). Cells grown to balanced growth were collected at OD 0.3-0.4 and transferred to the dormancy-inducing medium at a 1:1 dilution, such that the optical density at the beginning of the measurements was within the microplate readers sensitivity range $(\sim 0.2)$. Empty wells adjacent to the samples were filled with water to minimize evaporation. Plates were grown at $37^{\circ} \mathrm{C}$ with $700 \mathrm{rpm}$ shaking (double orbital mode) for $5 \mathrm{~h}$ with readings taken every $12 \mathrm{~min}$.

For fluorescence intensity comparisons (Fig. 3A and SI Fig. 5A) and for in vitro studies on cell lysates we did not grow cells to balanced growth. Instead, we inoculated single colonies in LB medium with $50 \mu \mathrm{g} / \mathrm{ml}$ of kanamycin and grew them overnight at $37^{\circ} \mathrm{C}$ with shaking at $220 \mathrm{rpm}$. We then inoculated an aliquot of the culture in RDM with glucose Brouwers et al. [2020], University Of Wisconsin (USA) [2020] and $50 \mu \mathrm{g} / \mathrm{ml}$ of kanamycin at a $1: 1000$ dilution and grew it at $37^{\circ} \mathrm{C}$ with shaking at $220 \mathrm{rpm}$ to OD 0.3-0.5.

\section{Fluorescence microscopy and motor speed measurements}

Imaging was carried out in a custom-built microscope with a 100x oil immersion objective lens (Nikon, Japan) Krasnopeeva et al. [2019], Rosko et al. [2017]. Illumination for the cells expressing pHluorin sensor was a neutral white LED with the filter ET470/40x (Chroma Technology, USA) and a UV LED (Cairn, UK). Cells expressing the Q7* sensor were illuminated with the same UV LED and a $488 \mathrm{~nm}$ laser (Vortran Laser Technology, USA). Images were taken with an iXon Ultra 897 EMCCD camera (Andor, UK) Krasnopeeva [2018], Rosko et al. [2017]. For both sensors, emission was measured with the ET525/40m filter (Chroma Technology, USA). In the case of pHluorin, images were taken at $1.5 \mathrm{~min}$ intervals, exposure time was $50 \mathrm{~ms}$ and Andor camera gain 100. Q7* was instead imaged only once per field of view with the same exposure time but with 50 Andor camera gain. Cells were imaged in a custom-built flow-cell (Mancini et al. [2020]), and attached to the coverslip surface as before Mancini et al. [2020]. Briefly, 1\% Poly-L-Lysine (Sigma, UK) were flushed through the flow cell and washed with 3-5 ml of growth media after $10 \mathrm{~s}$. Cells' flagellar filaments were sheared as before Krasnopeeva et al. [2019], Mancini et al. [2020], Rosko et al. [2017] by passing them through two syringes with narrow-gauge needles (26 gauge) connected by plastic tubing. $200 \mu \mathrm{l}$ of cells were delivered to the flow-cell and allowed to attach for $10 \mathrm{~min}$, after which the unattached cells were removed with $1 \mathrm{ml}$ of growth medium. Polystyrene particles (beads) with a diameter of $0.5 \mu \mathrm{m}$ (Polysciences, USA), were next delivered into the flow-cell and allowed to attach to the filament stubs. After 10 min unattached beads were removed with 1-2 ml of growth media. During measurements, fresh medium was constantly delivered with a peristaltic pump (Fusion 400, Chemyx, USA) using $100 \mu \mathrm{l} / \mathrm{min}$ flow rate. Cells grow attached to the Poly-L-Lysine surface with expected growth rates (given the medium), as previously reported Wang et al. [2019].

\section{QUEEN sensors characterization from lysates}

To characterise the sensors in their original conformation while maintaining conditions as similar as possible to the in vivo environment, we performed spectroscopic and microscopic assays on cell lysates. Cells grown in RDM were harvested via centrifugation at $11000 \mathrm{~g}$ at $4^{\circ} \mathrm{C}$ for $30 \mathrm{~min}$. Supernatant was discarded and cells resuspended in ice cold QUEEN buffer (50 mM HEPES, $200 \mathrm{mM} \mathrm{KCl}, 1 \mathrm{mM} \mathrm{MgCl} 2$, 
0.05\% Triton X-100, protease inhibitor cocktail (Sigma, GB), pH adjusted to 7.7) Yaginuma et al. [2014]. $100 \mu \mathrm{ug} / \mathrm{ml}$ of lysozyme was added to the cell suspension and incubated for $15 \mathrm{~min}$ at room temperature $\left(21^{\circ} \mathrm{C}\right)$. Cells were disrupted with a Soniprep 150 sonicator (MSE, UK) with 4 cycles of $30 \mathrm{~s}$ on and $30 \mathrm{~s}$ off, making sure that no foam was produced. During sonication the tube containing the sample was kept in ice cold water to limit protein damage. The lysates were then filtered with a $0.22 \mu \mathrm{m}$ filter to remove intact cells and large debris. Solutions with different concentrations of Mg-ATP (Sigma, UK) in QUEEN buffer were mixed with QUEEN lysate maintaining a constant ratio between the two (9:1) in order to maintain a constant protein concentration.

Samples for imaging were prepared in a tunnel slides Rosko et al. [2017] by flushing in sequence: $10 \mu 1$ 1\% Poly-L-Lysine (Sigma,UK), $100 \mu \mathrm{l}$ QUEEN buffer wash, $10 \mu \mathrm{l}$ of a 1:1 $1 \mu \mathrm{m}$ polystyrene bead solution (Polysciences, USA), $100 \mu 1$ QUEEN buffer wash, $10 \mu 1$ of QUEEN lysate and ATP solution. No incubation was needed between the different steps. The slides were then sealed with CoverGrip ${ }^{T M}$ sealant (Biotium, US). Three distant (at least 3 fields of view apart) fields of view were imaged with epifluorescence microscopy from each slide, the imaging plane was chosen by focusing on the polystyrene beads.

Fluorescence spectra of QUEEN sensors were obtained with a SPEX Fluoromax 3 spectrometer (Horiba, JP). Excitation was scanned from 370 to $500 \mathrm{~nm}$ at $513 \mathrm{~nm}$ emission with a slit size of $3 \mathrm{~nm}$. The temperature of the sample holder was controlled via a circulating water bath and readings were taken after letting the temperature of the sample reach equilibrium with that of the sample holder $\left(24^{\circ} \mathrm{C}\right)$, i.e. after at least $5 \mathrm{~min}$.

\section{MIC estimation}

MIC was estimated on EK07 cells grown to balanced growth. Aliquots of cultures in balanced growth were diluted 1:100 into pre-warmed M63 medium (final OD 0.002-0.004) with different concentrations of ciprofloxacin $(2,4,8,16,32,64,128,256 \mathrm{ng} / \mathrm{ml})$ or kanamycin $(2,4,8,16,32,64,128,256 \mu \mathrm{g} / \mathrm{ml})$.Cell suspensions were then transferred into a pre-warmed 96-well plate, incubated for $24 \mathrm{~h}$ and imaged (SI Fig. 10).

\section{CFU counting}

For CFU counting cells were grown to balanced growth, harvested via centrifugation at $8000 \mathrm{~g}$ for $5 \mathrm{~min}$ and washed twice in either M63 $+10 \mathrm{mM}$ indole, M63 $+200 \mu \mathrm{M}$ chloramphenicol, M63 deprived of the carbon source or fresh M63. Cultures were then incubated at $37^{\circ} \mathrm{C}$ with $220 \mathrm{rpm}$ shaking (the same conditions in which they had been grown). From this point onward the protocol varies slightly depending on whether we are trying to estimate survival to dormancy-inducing conditions (Fig. 1B) or to the combination of dormancy-inducing conditions and antibiotic treatments (Fig. 4).

For estimating the number of survivors to our dormancy-inducing conditions (Fig. 1B), aliquots of the cultures were taken after $60,120,180$ and $240 \mathrm{~min}$, pelleted at $8000 \mathrm{~g}$ for $2 \mathrm{~min}$ and washed twice in fresh M63 medium. Samples were then serially diluted with a 1:9 ratio in fresh M63 and $10 \mu 1$ of each dilution spotted on rectangular petri dishes (Thermo Fisher Scientific, UK) containing LB agar. The petri dishes were next tilted to let the liquid spread across the plate as in Jett et al. [1997], incubated for $24 \mathrm{~h}$ at $37^{\circ} \mathrm{C}$ and colonies counted.

For estimating survival to antibiotics (Fig. 4), cells were left in the dormancy-inducing conditions for $30 \mathrm{~min}$. Once dormancy had been established, antibiotics at $5 \mathrm{x}$ the MIC $(8 \mathrm{ng} / \mathrm{ml}$ for ciprofloxacin and $8 \mu / \mathrm{ml}$ for kanamycin (SI Fig. 10)) were added to the cultures. Aliquots of the cultures were taken after 30, 120, 180 and 240 min and plated as explained above.

Experiments were carried out in biological triplicates.

\section{Data analysis}

\section{Motor speed traces}

Raw traces of the $\mathrm{x}$ and $\mathrm{y}$ position of the bead attached to the filament stub were analyzed by a movingwindow discrete Fourier transform as in Rosko et al. [2017]. From the obtained motor speed traces DC frequency $(50 \mathrm{~Hz})$ was removed, speeds lower then $5 \mathrm{~Hz}$ ignored, and subsequently a median filter (window size 31) was applied Krasnopeeva et al. [2019], Mancini et al. [2020]. We use a chemotactic wild type strain for which the flagellar motor can change rotational direction, which appears as a negative speed after application of the moving-window Fourier transform. For the purpose of the PMF measurements 
these short intervals can be disregarded, and we only show the speed values above $0 \mathrm{~Hz}$ as in Mancini et al. [2020].

\section{Fluorescence images}

The image analysis was carried out with a custom written python script. Fluorescence images were initially inspected for unevenness of the field of view by assaying background greyvalues using the straight line tool of ImageJ Schneider et al. [2012]. To individuate the cells, objects with high grey values were discerned from the background by applying a global threshold via the Otsu's method Otsu [1979] and labeled. All found objects were inspected for size and aspect ratio so that anything smaller than $8 \mu \mathrm{m}^{2}$ or bigger than $56 \mu \mathrm{m}^{2}$ or with an aspect ratio below 1.7 were excluded. The criteria were set based on the average size, length and width of cells in our growth condition (balanced exponential growth at the growth rate of $0.7 \mathrm{~h}^{-1}$ ). Total cells' intensity values were obtained by summing up and averaging pixel intensities of selected objects.

\section{Plate reader data}

Curves from different repeats were averaged and the standard deviation estimated.

\section{Spectroscopy}

The intensity of the light source of the SPEX Fluoromax 3 spectrometer (Horiba, JP) across the range of excitation wavelength examined was measured during data acquisition and each fluorescence measurement obtained normalised by it, thus eliminating differences due to the light source. The results of the experiments, which were carried out in triplicate, were averaged and the standard deviation calculated. The spectra obtained were normalised by the average of the replicates' values at $435 \mathrm{~nm}$ excitation, which is the isosbestic point of the QUEEN spectrum, insensitive to ATP concentration.

\section{AUTHOR CONTRIBUTIONS}

L.M. and T.P. conceived the experiments. L.M. performed the experiments and analyzed experimental data. L.M. and T.P. interpreted the results and wrote the manuscript.

\section{ACKNOWLEDGEMENTS}

We are grateful to Hideyuki Yaginuma for donating us constructs containing the QUEEN sensors and for providing us the published data with QUEEN excitation spectrum. We are also grateful to Alessia Lepore and Meriem El Karoui for donating us an aliquot of ciprofloxacin and to members of Pilizota lab as well as Alessia Lepore, Meriem El Karoui, Ssu-Yuan Lin and Chien-Jung Lo for useful discussions.

This work was financially supported by the Cunningham Trust scholarship ACC/KWF/PhD1 to T.P. and L.M.. T.P. acknowledges the support of Human Frontier Science Program Grant (RGP0041/2015), and UK Research Councils Synthetic Biology for Growth program and is a member of the Biotechnology and Biological Sciences Research Council/Engineering and Physical Sciences Research Council/Medical Research Council-funded Synthetic Biology Research Centre (BB/M018040/1).

\section{COMPETING INTEREST}

None declared.

\section{REFERENCES}

Aedo, S. J., Orman, M. A., and Brynildsen, M. P. (2019). Stationary phase persister formation in escherichia coli can be suppressed by piperacillin and pbp3 inhibition. BMC microbiology, 19(1):140140. 31234796[pmid].

Anes, J., McCusker, M. P., Fanning, S., and Martins, M. (2015). The ins and outs of rnd efflux pumps in escherichia coli. Frontiers in Microbiology, 6:587.

Balaban, N. Q., Helaine, S., Lewis, K., Ackermann, M., Aldridge, B., Andersson, D. I., Brynildsen, M. P., Bumann, D., Camilli, A., Collins, J. J., Dehio, C., Fortune, S., Ghigo, J.-M., Hardt, W.-D., Harms, A., Heinemann, M., Hung, D. T., Jenal, U., Levin, B. R., Michiels, J., Storz, G., Tan, M.-W., Tenson, T., Van Melderen, L., and Zinkernagel, A. (2019). Definitions and guidelines for research on antibiotic persistence. Nature Reviews Microbiology, 17(7):441-448. 
Bearne, S. L. (2014). Illustrating the effect of ph on enzyme activity using gibbs energy profiles. Journal of Chemical Education, 91(1):84-90.

Beloin, C., Roux, A., and Ghigo, J. M. (2008). Escherichia coli biofilms. Current topics in microbiology and immunology, 322:249-289. 18453280[pmid].

Blanco, P., Hernando-Amado, S., Reales-Calderon, J. A., Corona, F., Lira, F., Alcalde-Rico, M., Bernardini, A., Sanchez, M. B., and Martinez, J. L. (2016). Bacterial multidrug efflux pumps: Much more than antibiotic resistance determinants. Microorganisms, 4(1):14. 27681908[pmid].

Bradbeer, C. (1993). The proton motive force drives the outer membrane transport of cobalamin in Escherichia coli. Journal of bacteriology, 175(10):3146-50.

Braetz, S., Schwerk, P., Thompson, A., Tedin, K., and Fulde, M. (2017). The role of atp pools in persister cell formation in (fluoro)quinolone-susceptible and -resistant strains of salmonella enterica ser. typhimurium. Veterinary Microbiology, 210:116 - 123.

Brauner, A., Fridman, O., Gefen, O., and Balaban, N. Q. (2016). Distinguishing between resistance, tolerance and persistence to antibiotic treatment. Nature Reviews Microbiology, 14:320 EP -.

Brouwers, R., Vass, H., Dawson, A., Squires, T., Tavaddod, S., and Allen, R. J. (2020). Stability of -lactam antibiotics in bacterial growth media. bioRxiv.

Chimerel, C., Field, C. M., Piñero-Fernandez, S., Keyser, U. F., and Summers, D. K. (2012). Indole prevents escherichia coli cell division by modulating membrane potential. Biochimica et biophysica acta, 1818(7):1590-1594. 22387460[pmid].

Conlon, B. P., Rowe, S. E., Gandt, A. B., Nuxoll, A. S., Donegan, N. P., Zalis, E. A., Clair, G., Adkins, J. N., Cheung, A. L., and Lewis, K. (2016). Persister formation in staphylococcus aureus is associated with atp depletion. Nature microbiology, 1:16051.

Coussens, N. P. and Daines, D. A. (2016). Wake me when it's over - bacterial toxin-antitoxin proteins and induced dormancy. Experimental biology and medicine (Maywood, N.J.), 241(12):1332-1342. 27216598[pmid].

Elowitz, M. B., Surette, M. G., Wolf, P.-E., Stock, J., and Leibler, S. (1997). Photoactivation turns green fluorescent protein red. Current Biology, 7(10):809-812.

Fung, D. C. and Berg, H. C. (1995). Powering the flagellar motor of escherichia coli with an external voltage source.

Gabel, C. V. and Berg, H. C. (2003). The speed of the flagellar rotary motor of escherichia coli varies linearly with protonmotive force. Proceedings of the National Academy of Sciences, 100(15):87488751.

Gale, E. F. and Folkes, J. P. (1953). The assimilation of amino-acids by bacteria. xv. actions of antibiotics on nucleic acid and protein synthesis in staphylococcus aureus. The Biochemical journal, 53(3):493498. $13032100[\mathrm{pmid}]$.

Gefen, O., Gabay, C., Mumcuoglu, M., Engel, G., and Balaban, N. Q. (2008). Single-cell protein induction dynamics reveals a period of vulnerability to antibiotics in persister bacteria. Proceedings of the National Academy of Sciences, 105(16):6145-6149.

Gout, E., Rébeillé, F., Douce, R., and Bligny, R. (2014). Interplay of mg2+, adp, and atp in the cytosol and mitochondria: Unravelling the role of $\mathrm{mg} 2+$ in cell respiration. Proceedings of the National Academy of Sciences, 111(43):E4560-E4567.

Grant, S. S. and Hung, D. T. (2013). Persistent bacterial infections, antibiotic tolerance, and the oxidative stress response. Virulence, 4(4):273-283. 23563389[pmid].

Grassi, L., Di Luca, M., Maisetta, G., Rinaldi, A. C., Esin, S., Trampuz, A., and Batoni, G. (2017). Generation of persister cells of pseudomonas aeruginosa and staphylococcus aureus by chemical treatment and evaluation of their susceptibility to membrane-targeting agents. Frontiers in Microbiology, 8:1917.

Händel, N., Schuurmans, J. M., Brul, S., and ter Kuile, B. H. (2013). Compensation of the metabolic costs of antibiotic resistance by physiological adaptation in escherichia coli. Antimicrobial agents and chemotherapy, 57(8):3752-3762. 23716056[pmid].

Harvey, R. J. and Koch, A. L. (1980). How partially inhibitory concentrations of chloramphenicol affect the growth of escherichia coli. Antimicrobial agents and chemotherapy, 18(2):323-337. 6160809[pmid].

Jahreis, K., Pimentel-Schmitt, E. F., Brückner, R., and Titgemeyer, F. (2008). Ins and outs of glucose transport systems in eubacteria. FEMS Microbiology Reviews, 32(6):891-907.

Jelenc, P. C. and Kurland, C. G. (1984). Multiple effects of kanamycin on translational accuracy. 
Molecular and General Genetics MGG, 194(1):195-199.

Jett, B. D., Hatter, K. L., Huycke, M. M., and Gilmore, M. S. (1997). Simplified agar plate method for quantifying viable bacteria. BioTechniques, 23(4):648-650. PMID: 9343684.

Kim, J. and Park, W. (2015). Indole: a signaling molecule or a mere metabolic byproduct that alters bacterial physiology at a high concentration? Journal of Microbiology, 53(7):421-428.

Kim, M., Zhang, Z., Okano, H., Yan, D., Groisman, A., and Hwa, T. (2012). Need-based activation of ammonium uptake in escherichia coli. Molecular Systems Biology, 8(1):616.

Kowalczykowski, S. (1991). Biochemistry of genetic recombination: energetics and mechanism of dna strand exchange. Annual review of biophysics and biophysical chemistry, 20:539—575.

Kowalczykowski, S. C., Dixon, D. A., Eggleston, A. K., Lauder, S. D., and Rehrauer, W. M. (1994). Biochemistry of homologous recombination in escherichia coli. Microbiological reviews, 58(3):401465. 7968921[pmid].

Krasnopeeva, E. (2018). Single cell measurements of bacterial physiology traits during exposure to an external stress. PhD thesis, University of Edinburgh.

Krasnopeeva, E., Lo, C.-J., and Pilizota, T. (2019). Single-cell bacterial electrophysiology reveals mechanisms of stress-induced damage. Biophysical Journal, 116(12):2390 - 2399.

Kreuzer, K. N. (2013). Dna damage responses in prokaryotes: regulating gene expression, modulating growth patterns, and manipulating replication forks. Cold Spring Harbor perspectives in biology, 5(11):a012674-a012674. 24097899[pmid].

Lazazzera, B. A. (2000). Quorum sensing and starvation: signals for entry into stationary phase. Current Opinion in Microbiology, 3(2):177 - 182.

Leszczynska, D., Matuszewska, E., Kuczynska-Wisnik, D., Furmanek-Blaszk, B., and Laskowska, E. (2013). The formation of persister cells in stationary-phase cultures of escherichia coli is associated with the aggregation of endogenous proteins. PLOS ONE, 8(1):1-10.

Levin-Reisman, I., Ronin, I., Gefen, O., Braniss, I., Shoresh, N., and Balaban, N. Q. (2017). Antibiotic tolerance facilitates the evolution of resistance. Science, 355(6327):826-830.

Lewis, K. (2007). Persister cells, dormancy and infectious disease. Nature Reviews Microbiology, $5(1): 48-56$.

Liu, J., Gefen, O., Ronin, I., Bar-Meir, M., and Balaban, N. Q. (2020). Effect of tolerance on the evolution of antibiotic resistance under drug combinations. Science, 367(6474):200-204.

Maaløe, O., . K. N. O. (1966). Control of macromolecular synthesis: A study of DNA, RNA, and protein synthesis in bacteria. New York: W.A. Benjamin.

Mancini, L., Terradot, G., Tian, T., Pu, Y., Li, Y., Lo, C.-J., Bai, F., and Pilizota, T. (2020). A general workflow for characterization of nernstian dyes and their effects on bacterial physiology. Biophysical Journal, 118(1):4 - 14 .

Mitchell, P. (1961). Coupling of phosphorylation to electron and hydrogen transfer by a chemi-osmotic type of mechanism. Nature, 191:144.

Otsu, N. (1979). A threshold selection method from gray-level histograms. IEEE Transactions on Systems, Man, and Cybernetics, 9(1):62-66.

Patterson, G. H. and Lippincott-Schwartz, J. (2002). A photoactivatable gfp for selective photolabeling of proteins and cells. Science, 297(5588):1873-1877.

Piddock, L. J. V. (2006). Clinically relevant chromosomally encoded multidrug resistance efflux pumps in bacteria. Clinical microbiology reviews, 19(2):382-402. 16614254[pmid].

Pilizota, T. and Shaevitz, J. W. (2012). Fast, multiphase volume adaptation to hyperosmotic shock by escherichia coli. PloS one, 7(4):e35205-e35205.

Pletnev, P., Osterman, I., Sergiev, P., Bogdanov, A., and Dontsova, O. (2015). Survival guide: Escherichia coli in the stationary phase. Acta naturae, 7(4):22-33. 26798489[pmid].

Ramos, S. and Kaback, H. R. (1977). The relation between the electrochemical proton gradient and active transport in escherichia coli membrane vesicles. Biochemistry, 16(5):854-859.

Rittershaus, E., Baek, S.-H., and Sassetti, C. (2013). The normalcy of dormancy: Common themes in microbial quiescence. Cell Host Microbe, 13(6):643 - 651.

Ronneau, S. and Helaine, S. (2019). Clarifying the link between toxin-antitoxin modules and bacterial persistence. Journal of Molecular Biology, 431(18):3462 - 3471. The molecular basis of antibiotic action and resistance.

Rosko, J., Martinez, V. A., Poon, W. C. K., and Pilizota, T. (2017). Osmotaxis in escherichia coli through 
changes in motor speed. Proceedings of the National Academy of Sciences.

Ryu, W. S., Berry, R. M., and Berg, H. C. (2000). Torque-generating units of the flagellar motor of escherichia coli have a high duty ratio. Nature, 403:444.

Schneider, C. A., Rasband, W. S., and Eliceiri, K. W. (2012). Nih image to imagej: 25 years of image analysis. Nature Methods, 9(7):671-675.

Shan, Y., Brown Gandt, A., Rowe, S. E., Deisinger, J. P., Conlon, B. P., and Lewis, K. (2017). Atpdependent persister formation in escherichia coli. mBio, 8(1).

Stevenson, K., McVey, A. F., Clark, I. B. N., Swain, P. S., and Pilizota, T. (2016). General calibration of microbial growth in microplate readers. Scientific Reports, 6:38828 EP -. Article.

Svenningsen, M. S., Veress, A., Harms, A., Mitarai, N., and Semsey, S. (2019). Birth and resuscitation of (p)ppgpp induced antibiotic tolerant persister cells. Scientific Reports, 9(1):6056.

Taber, H. W., Mueller, J. P., Miller, P. F., and Arrow, A. S. (1987). Bacterial uptake of aminoglycoside antibiotics. Microbiological reviews, 51(4):439-457.

Tran, T., Ran, Q., Ostrer, L., and Khodursky, A. (2016). De novo characterization of genes that contribute to high-level ciprofloxacin resistance in escherichia coli. Antimicrobial Agents and Chemotherapy, 60(10):6353-6355.

University Of Wisconsin (USA) (2020). Rich defined medium protocol. https: / /www . genome . wisc.edu/resources/protocols/ezmedium.htm.

Wang, Y.-K., Krasnopeeva, E., Lin, S.-Y., Bai, F., Pilizota, T., and Lo, C.-J. (2019). Comparison of escherichia coli surface attachment methods for single-cell microscopy. Scientific Reports, 9(1):19418.

Wood, J. M. (2015). Bacterial responses to osmotic challenges. The Journal of General Physiology, 145(5):381-388.

Yaginuma, H., Kawai, S., Tabata, K. V., Tomiyama, K., Kakizuka, A., Komatsuzaki, T., Noji, H., and Imamura, H. (2014). Diversity in atp concentrations in a single bacterial cell population revealed by quantitative single-cell imaging. Scientific Reports, 4:6522 EP -.

Zeiler, H. J. (1985). Evaluation of the in vitro bactericidal action of ciprofloxacin on cells of escherichia coli in the logarithmic and stationary phases of growth. Antimicrobial agents and chemotherapy, 28(4):524-527. 2934022[pmid]. 